\title{
Progression of cardiovascular and endocrine dysfunction in a rabbit model of obesity
}

\begin{abstract}
Gabriela A Eppel ${ }^{1}$, James A Armitage ${ }^{2,3}$, Nina Eikelis ${ }^{3}$, Geoffrey A Head ${ }^{3}$ and Roger G Evans ${ }^{1}$
In rabbits, mean arterial pressure (MAP) increases in response to fat feeding, but does not increase further with progressive weight gain. We documented the progression of adiposity and the alterations in endocrine/cardiovascular function in response to fat feeding in rabbits, to determine whether stabilization of MAP after 3 weeks could be explained by stabilization of neurohormonal factors. Rabbits were fed a control diet or high-fat diet for 9 weeks $(n=23)$. Fat feeding progressively increased body mass and adiposity. Heart rate (HR) was elevated by week $3(15 \pm 3 \%)$ but changed little thereafter. The effects of fat feeding on MAP were dependent on baseline MAP and peaked at 3 weeks. From baseline, MAP $\leqslant 80 \mathrm{~mm} \mathrm{Hg}$, MAP had increased by $8.1 \pm 1.3,4.7 \pm 1.7$ and $5.6 \pm 1.2 \mathrm{~mm} \mathrm{Hg}$, respectively, 3,6 and 9 weeks after commencing the high-fat diet, but by only $2.6 \pm 1.5,3.0 \pm 1.7$ and $3.9 \pm 1.4 \mathrm{~mm} \mathrm{Hg}$, respectively, in control rabbits. Fat feeding did not increase MAP from a baseline $>80 \mathrm{~mm} \mathrm{Hg}$. Plasma concentrations of leptin and insulin increased during the first 3-6 weeks of fat feeding and then stabilized (increasing by $111 \pm 17 \%$ and $731 \pm 302 \%$ by week 9 , respectively), coinciding with the pattern of changes in MAP and HR. Plasma total cholesterol, triglycerides, renin activity, aldosterone and atrial natriuretic peptide were not significantly altered by fat feeding. Given that the changes in plasma leptin and insulin mirrored the changes in MAP and HR, leptin and insulin may be important factors in the development of hypertension and tachycardia in the rabbit model of obesity. Hypertension Research (2013) 36, 588-595; doi:10.1038/hr.2013.2; published online 14 February 2013
\end{abstract}

Keywords: atrial natriuretic peptide; dual-energy X-ray absorptometry; insulin; leptin; renin-angiotensin-aldosterone system

\section{INTRODUCTION}

It has been argued that obesity represents the single-most important cause of essential hypertension. ${ }^{1}$ The mechanistic link between obesity and hypertension is thought to chiefly involve excessive secretion of leptin from adipocytes, which in turn leads to activation of the sympathetic nervous system and downstream events such as increased heart rate (HR), vascular resistance and renal salt and water retention. ${ }^{1}$ Vagal withdrawal probably also contributes to the tachycardic effect of fat feeding. ${ }^{2,3}$ There is also evidence that leptin has a range of peripheral effects on the structure and function of the kidney, heart and blood vessels, which can affect long-term blood pressure control. ${ }^{1}$ Other factors, such as hyperinsulinaemia, reduced circulating levels of adiponectin, ${ }^{4}$ activation of the renin-angiotensinaldosterone system, inflammatory cytokine pathways and oxidative stress are also thought to contribute. ${ }^{5}$

The rabbit has been extensively used as a model of obesity. Numerous reports have documented studies in which feeding rabbits a high-fat diet, for periods ranging from 2-12 weeks, results in increased adiposity, associated with a $10-20 \%$ increase in mean arterial pressure (MAP) and a $10-35 \%$ increase in $\mathrm{HR}^{2,6-16}$ Telemetric recordings in the fat-fed rabbit have shown that elevations in MAP and HR occur quickly, within days of commencing fat feeding, even before major changes in body weight occur. ${ }^{9,17}$ Furthermore, increases in MAP and HR are progressive, within a 3-week time frame, but then seem to plateau. ${ }^{9,17}$ This is in contrast with observations in humans where the degree of hypertension is proportional to body mass index or other indices of adiposity. ${ }^{18,19}$ In humans, the neurohormonal abnormalities associated with obesity, such as hyperleptinaemia, also increase progressively relative to the degree of adiposity. ${ }^{20-22}$ We know that in the rabbit, plasma leptin and insulin are elevated within the first few weeks of fat feeding, ${ }^{17}$ but whether they continue to increase progressively, in line with body weight and adiposity, remains to be determined. The current study was therefore designed to explore the outcomes of 9 weeks of fat feeding. We documented the changes in adiposity, MAP and HR, plasma leptin and other hormonal factors. We hypothesized that the absence of progressive increases in MAP in rabbits, beyond the first 3 weeks of fat feeding, can be accounted for by the absence of progressive neurohormonal abnormalities. This hypothesis predicts that plasma levels of leptin and insulin stabilize after 3 weeks of fat feeding, despite continued increases in adiposity.

Previous anecdotal observations indicate that rabbits with relatively high MAP ( $>80 \mathrm{~mm} \mathrm{Hg})$, measured via the ear artery at baseline, often do not have a marked pressor response to chronic fat feeding

${ }^{1}$ Department of Physiology, Monash University, Melbourne, Victoria, Australia; ${ }^{2}$ Department of Anatomy and Developmental Biology, Monash University, Melbourne, Victoria, Australia and ${ }^{3}$ Baker IDI Heart and Diabetes Institute, Melbourne, Victoria, Australia

Correspondence: Dr RG Evans, Department of Physiology, PO Box 13F, Monash University, Victoria 3800, Australia.

E-mail: roger.evans@monash.edu

Received 13 August 2012; revised 29 November 2012; accepted 4 December 2012; published online 14 February 2013 
(Head et al., unpublished observations). Therefore, we also took the opportunity to test the hypothesis that the response of MAP to fat feeding in rabbits depends in part on baseline MAP.

\section{METHODS}

\section{Animals}

A total of 46 male New Zealand white rabbits $(3.2 \pm 0.1 \mathrm{~kg}, 16 \pm 1$ weeks old $)$ were sourced from Nanowie Small Animal Production Unit, Modewarre, Victoria, Australia. The rabbits were studied according to the Australian Code of Practice for the Care and Use of Animals for Scientific Purposes and the protocol was approved in advance by the Animal Ethics Committee of the Monash University School of Biomedical Sciences. Data from a subset of these rabbits have been be reported elsewhere. ${ }^{23}$ However, the previous report focused on data collected after the conclusion of the current experiments, so any overlap is minor and detailed below.

\section{Experimental protocol}

After a 2-week acclimatization period, during which they consumed a control diet, the rabbits were randomly assigned to receive either a high-fat diet or the control diet for 9 weeks ( $n=23$ per group). The control diet was a standard rabbit pellet formulation containing $\sim 4 \%$ fat derived from vegetable oils (Specialty Feeds, Glen Forrest, Western Australia, Australia; Table 1). Rabbits on the control diet were given $100 \mathrm{~g}$ of pellets per day. The high-fat diet was a custom-made variation of the standard pellet formulation with $\sim 14 \%$ fat derived from vegetable and animal sources (5\% soya oil and 5\% lard added, Specialty Feeds). Rabbits on the high-fat diet were given $150 \mathrm{~g}$ of pellets per

Table 1 Composition of the diets

\begin{tabular}{lcc}
\hline Components & Control diet & High-fat diet \\
\hline Fat (\%) & 4 & 14 \\
Protein (\%) & 18 & 17 \\
Crude fiber (\%) & 14 & 13 \\
Digestible energy $\left(\mathrm{MJ} \mathrm{kg}^{-1}\right)$ & 11 & 14 \\
\hline
\end{tabular}

day, although they did not always consume this total amount. Throughout the dietary intervention, body mass and food intake were determined on a weekly basis. MAP and HR were measured at 3-week intervals in conscious rabbits for a period of at least $1 \mathrm{~h}$ each time. The measurements were made via acute catheterization of a central ear artery under local analgesia (2.5\% lignocaine and 2.5\% prilocaine; Emla 5\% Cream; AstraZeneca; North Ryde, New South Wales, Australia followed by $1 \%$ lignicaine s.c.; Xylocaine; AstraZeneca), as described previously. ${ }^{24} \mathrm{MAP}$ and HR, measured in a subset of these animals at the 9-week time point, have been presented elsewhere. ${ }^{23}$ Blood samples $(\sim 7 \mathrm{ml})$ were collected immediately after measurement of arterial pressure. In a subset of animals, body composition was also determined at 3-week intervals by dual-energy X-ray absorptometry (DEXA; Discovery A; Hologic, Bedford, MA, USA). DEXA was performed under acute anesthesia with $1 \%$ propofol (Propofol Sandoz; Sandoz; Pyrmont, New South Wales, Australia) given via a marginal ear vein (given i.v. to effect). At the conclusion of the protocol, the rabbits entered an additional acute experiment, in which they were studied under pentobarbitone anaesthesia, which is described elsewhere. ${ }^{23}$ At the conclusion of this additional experiment rabbits were killed with an overdose of pentobarbitone given i.v. (Lethabarb, Virbac Australia, Milperra, New South Wales, Australia). Postmortem wet weight of the fat pads, heart, left ventricle and left kidney were determined and are reported herein.

\section{Blood sample analysis}

Plasma glucose concentration was measured immediately after blood collection using a glucometer (Optium Xceed Monitor, Abbott Australasia, Doncaster, Victoria, Australia). Blood samples were collected into chilled tubes containing Li-heparin for measurements of electrolytes, creatinine and aldosterone, into chilled tubes containing EDTA for measurement of insulin, leptin, cholesterol and triglycerides, into chilled tubes containing EDTA plus 2,3-dimercapto-1propanol for measurement of plasma renin activity (PRA), and into chilled tubes containing EDTA plus aprotinin (Sigma, St Louis, MO, USA) for measurement of atrial natriuretic peptide (ANP). The samples were centrifuged at $3000 \mathrm{~g}$ at $4{ }^{\circ} \mathrm{C}$ and the plasma removed for storage at $-80^{\circ} \mathrm{C}$ until analysis.

Electrolytes, creatinine, cholesterol and triglycerides were measured on a Clinical Analyzer (Beckman Coulter Australia, Gladesville, New South Wales, Australia). Immunoassays were used to measure aldosterone (Aldosterone Count-a-Coat RIA kit, Bio-Mediq DPC, Doncaster, Victoria, Australia) and
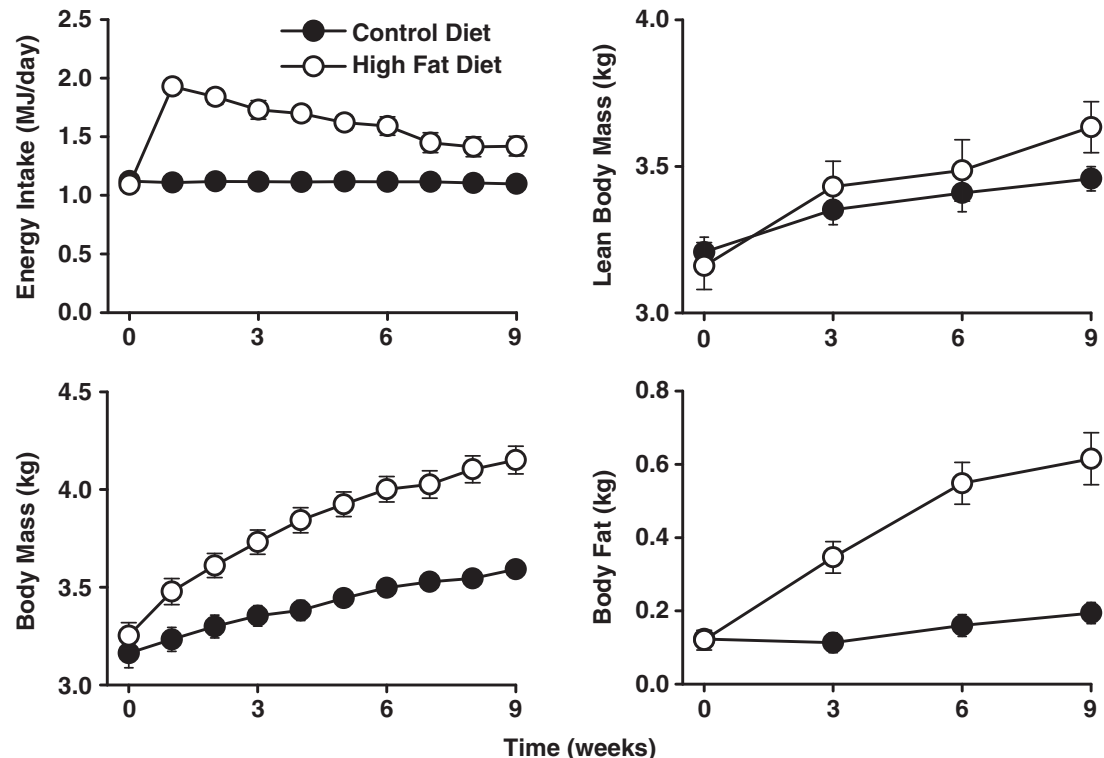

Figure 1 Impact of the 9-week diet protocol on energy intake, body mass, lean body mass and body fat. Lean body mass and body fat were determined by dual-energy X-ray absorptometry. Co-ordinates represent mean \pm s.e.m. of $n=23$ per group for caloric intake and body mass and $n=8$ (high-fat diet) and 9 (control diet) for lean body mass and body fat. Outcomes of repeated measures analysis of variance are shown in Table 2. 
ANP (ProSearch International Australia in-house RIA for human alpha ANP 1-28, Malvern, Victoria, Australia), insulin (Crystal Chem, Downers Grove, IL, USA), and leptin (Multispecies Leptin RIA kit, Linco Research Inc, St. Charles, MO, USA). PRA was measured by a method based on that of Johnston et al. ${ }^{25}$ (Prosearch International Australia). ${ }^{26}$ The quantitative insulin sensitivity check index (QUICKI) was used for assessment of insulin sensitivity. ${ }^{27}$

\section{Statistical methods}

Data are presented as mean \pm s.e.m. and two-tailed $P \leqslant 0.05$ was considered to be statistically significant. Serial measurements over the 9-week fat-feeding regimen were subjected to repeated measures analysis of variance, ${ }^{28}$ the factors comprising diet (control or high fat), time and in some cases subgroup (baseline MAP $\leqslant$ or $>80 \mathrm{mmHg}$ ). The relationships between the various measurements we made were assessed by linear regression. Lines of best fit were determined by the ordinary least-products method, to account for presence of experimental error in both the dependent and independent variables. ${ }^{29}$ Student's unpaired $t$-test ${ }^{30}$ was used for comparisons of baseline levels between subgroups based on baseline MAP and for comparison of postmortem measurements between fat-fed and lean rabbits.

\section{RESULTS}

Food intake, body weight and body composition

During the 9-week diet protocol, rabbits on the high-fat diet had a significantly greater energy intake than their counterparts on the control diet (Figure 1; Table 2). The total intake of the fat-fed rabbits was $103 \pm 3 \mathrm{MJ}$ over the course of the 9 weeks, compared with only $70 \pm 1 \mathrm{MJ}$ in the control group. This resulted in rabbits on the high-fat diet having a significantly greater increase in body mass than those on the control diet $(0.90 \pm 0.05$ vs. $0.43 \pm 0.06 \mathrm{~kg})$. The effects of the high-fat diet on food intake and weight gain were indistinguishable in rabbits with a baseline MAP $\leqslant 80 \mathrm{~mm} \mathrm{Hg}$ compared with those with a baseline MAP $>80 \mathrm{~mm} \mathrm{Hg}$ $\left(P_{\text {diet }} \times\right.$ time $\times$ subgroup $\geqslant 0.52$; data not shown $)$. More than half of the increased body mass in the fat-fed group was due to fat deposition as determined by DEXA $(0.50 \pm 0.07 \mathrm{~kg} v s .0 .07 \pm 0.02 \mathrm{~kg}$ in the control group). The increases in body mass and body fat were progressive over the course of the 9 weeks of fat feeding. The fat-fed group also had a greater increase in lean body mass than the control group $(0.47 \pm 0.06$ vs. $0.25 \pm 0.03 \mathrm{~kg})$.

\section{Arterial pressure and HR in conscious rabbits}

At baseline, 9 of the 23 rabbits randomized to remain on the control diet, and 11 of the 23 rabbits randomized to commence the high-fat diet, had a MAP $\leqslant 80 \mathrm{~mm} \mathrm{Hg}$ (mean $77.1 \pm 0.8 \mathrm{~mm} \mathrm{Hg}, n=20$ ). Thus, 14 rabbits randomized to the control diet and 12 rabbits randomized to the high-fat diet had a MAP $>80 \mathrm{~mm} \mathrm{Hg}$ (mean $86.6 \pm 1.0 \mathrm{~mm} \mathrm{Hg}$, $n=26$ ). HR tended to be lower in rabbits with a baseline MAP $\leqslant 80$ $\mathrm{mmHg}(171 \pm 5$ beats per minute) than those with a baseline MAP $>80 \mathrm{~mm} \mathrm{Hg}(183 \pm 4$ beats per minute), but this apparent effect was not statistically significant $(P=0.07$, unpaired $t$-test).

When MAP was analyzed across all rabbits, regardless of its baseline level, it was not significantly different between the two groups when assessed over the 9-week protocol (Figure 2; Table 2). Nevertheless, there was an increase in MAP from week 0 to week $3(+4.4 \pm 1.2$ $\mathrm{mm} \mathrm{Hg}, P=0.002$ by paired $t$-test) in the fat-fed group that was not observed in the control group (Figure 2). This increase was not sustained during the rest of the 9-week protocol. In contrast, HR was significantly elevated, by an average of $15 \pm 3 \%$, from weeks $3-9$ in the fat-fed group. HR remained relatively stable over the course of the experiment in the control group $(-3 \pm 2 \%)$.

In rabbits with a baseline $\mathrm{MAP} \leqslant 80 \mathrm{mmHg}$, MAP increased by $8.1 \pm 1.3,4.7 \pm 1.7$ and $5.6 \pm 1.2 \mathrm{~mm} \mathrm{Hg}$, respectively, at 3,6 and

Table 2 Outcomes of repeated measures analysis of variance for data in Figures 1-7

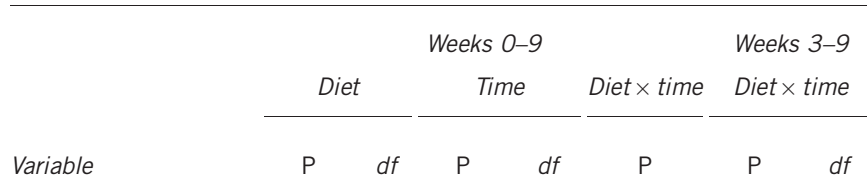

Figure 1

Energy intake

Body weight

Body fat

Lean body mass

\begin{tabular}{|c|c|c|c|}
\hline$<0.0011,44<0.001$ & 9,396 & $<0.001$ & 0.002 \\
\hline$<0.0011,44<0.00$ & ,396 & $<0.001$ & $<0.001$ \\
\hline$<0.0011,15<0.001$ & 3,45 & $<0.001$ & 0.05 \\
\hline $1,15<0.001$ & 3,45 & 0.01 & 0.15 \\
\hline
\end{tabular}

Figure 2

Mean arterial

pressure

Heart rate

$\begin{array}{lllll}0.23 & 1,44 & 0.01 & 3,132 & 0.21\end{array}$

$0.48 \quad 2,88$

Figure 3

Plasma glucose

Plasma insulin

$0.23 \quad 1,33 \quad 0.52 \quad 3,99$

0.07

$\begin{array}{lllll}0.01 & 1,12 & 0.006 & 3,36 & 0.02\end{array}$

$0.152,66$

$0.24 \quad 2,24$

Figure 4

Plasma leptin

$<0.001 \quad 1,40 \quad 0.02 \quad 3,120<0.001$

$0.27 \quad 2,70$

Figure 5

Total cholesterol

$0.591,40<0.0013,120$

$\begin{array}{llll}0.09 & 1,41 & 0.44 & 3,123\end{array}$

0.87

$0.662,80$

Triglycerides

Figure 6

Plasma renin activity

Plasma aldosterone

$\begin{array}{llll}0.48 & 1,19 & 0.35 & 3,57\end{array}$

0.81

$\begin{array}{llll}1.0 & 1,11 & 0.30 & 3,33\end{array}$

$\begin{array}{llll}0.04 & 1,11 & 0.79 & 3,33\end{array}$

0.71

0.68

Plasma atrial

Natriuretic peptide

Figure 7

Plasma sodium

Plasma potassium

$\begin{array}{llll}0.04 & 1,11 & 0.50 & 3,33\end{array}$

0.04

$\begin{array}{llll}0.45 & 1,11 & 0.39 & 3,33\end{array}$

$0.06 \quad 1,11 \quad 0.62 \quad 3,33$

0.07

0.06

$\begin{array}{lllll}0.17 & 1,11 & 0.29 & 3,33 & 0.09\end{array}$

$0.44 \quad 2,22$

$0.112,22$

$0.50 \quad 2,22$

Plasma chloride

$P$-values for within-subjects factors were conservatively adjusted by the Greenhouse-Geiss method. ${ }^{27}$ Significant $P$-values are in bold font. The degrees of freedom (df) differ according to variable because some analyses were performed on only a subset of animals. The $\mathrm{df}$ for the diet $\times$ time interaction are as for the main effect of time.

9 weeks after commencing the high-fat diet. In contrast, MAP increased by only $2.6 \pm 1.5,3.0 \pm 1.7$ and $3.9 \pm 1.4 \mathrm{~mm} \mathrm{Hg}$, respectively, across the same time period in rabbits on the control $\operatorname{diet}\left(P_{\text {diet }}=0.015\right)$. In rabbits with a baseline $\mathrm{MAP}>80 \mathrm{~mm} \mathrm{Hg}$, there were slight falls in MAP across the 9 weeks of the experiment which were indistinguishable in rabbits placed on the two diets $\left(P_{\text {diet }}=0.28\right.$; Figure 2$)$. The effects of fat feeding on changes in HR across the 9 weeks of the dietary intervention were indistinguishable in the two subgroups of rabbits with differing baseline MAP (Figure 2).

\section{Plasma glucose, insulin and leptin concentrations}

Plasma glucose was not significantly different between the two groups, although there was a tendency for plasma glucose to be elevated at the 3 -week time point in the fat-fed group (Figure 3; Table 2). 
We observed elevated plasma insulin concentrations from week 3 in the fat-fed group (Figure 3; Table 2) but the concentration remained relatively stable from weeks 3-9. QUICKI changed little across the 9 weeks of the dietary intervention in control animals (average over the 9 weeks $=0.214 \pm 0.004$ ). In contrast, QUICKI fell from $0.222 \pm 0.005$ to $0.189 \pm 0.004$ after 3 weeks of fat feeding, and thereafter changed little.

Plasma leptin concentration also increased significantly in the fatfed group, by $1.88 \pm 0.20 \mathrm{ng} \mathrm{ml}^{-1}$ from week 0 to week 6 , but changed little thereafter. In contrast, plasma leptin was little altered across the course of the 9 weeks of the study in the control group $\left(+0.23 \pm 0.16 \mathrm{ng} \mathrm{ml}^{-1}\right.$, Figure $\left.4 \mathrm{a}\right)$. The effects of fat feeding on plasma leptin concentration were indistinguishable in the two subgroups of rabbits with differing baseline MAP (data not shown). When data from all rabbits and time points were combined, there was a robust relationship between fat mass and plasma leptin concentration (Figure $4 \mathrm{~b}$ ). However, most of the data points from the fat-fed group at weeks 6 and 9, are fairly equally distributed around the $4 \mathrm{ng} \mathrm{ml}^{-1}$ level. This reflects a lack of progression of hyperleptinaemia beyond week 6 .

\section{Plasma lipids}

Plasma total cholesterol concentration progressively reduced across the 9 weeks of the dietary intervention but did not differ significantly between control and fat-fed rabbits (Figure 5; Table 2). There was a tendency for triglyceride concentrations to be elevated in the fat-fed group, but this apparent effect did not reach statistical significance (Figure 5; Table 2).

\section{PRA and aldosterone and ANP concentrations}

In rabbits receiving the control diet, PRA and plasma ANP remained relatively stable across the 9 weeks of the experiment. Plasma aldosterone concentration appeared to progressively reduce over the 9-week course of the experiment, but this trend was not statistically significant (Figure 6; Table 2). Plasma ANP was slightly but significantly less in fat-fed rabbits than controls across the 9 weeks of the experiment, but fat feeding did not significantly alter the profile of levels of PRA or plasma aldosterone (Figure 6; Table 2).
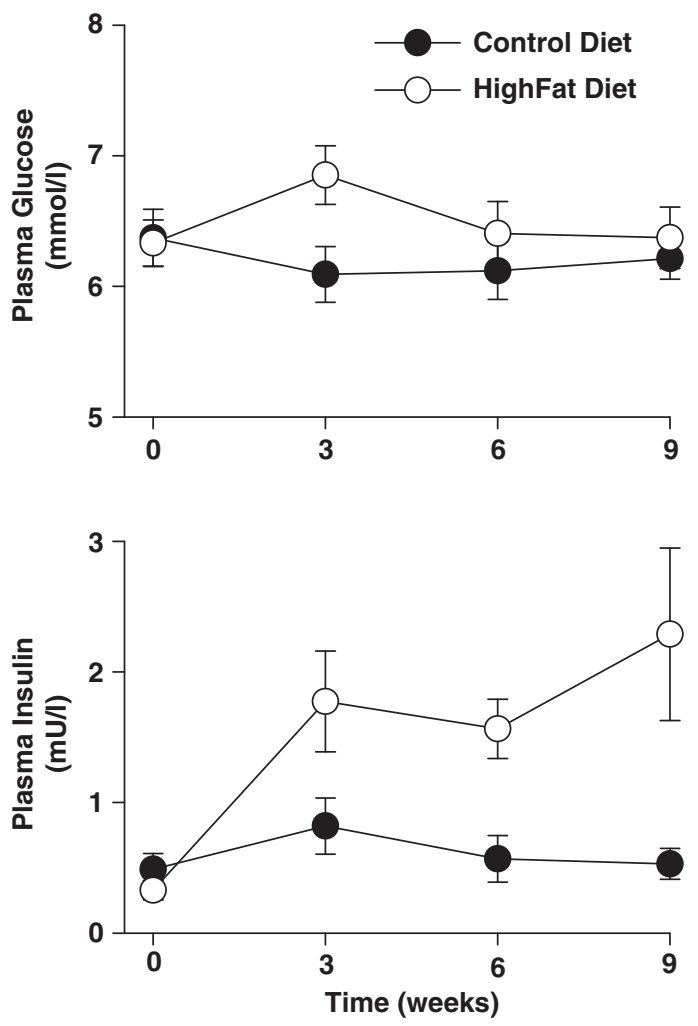

Figure 3 Plasma glucose and insulin concentrations across the 9-week dietary intervention. Co-ordinates represent mean \pm s.e.m. of $n=17$ (control diet) and 18 (high-fat diet) for plasma glucose and $n=7$ per group for insulin. Outcomes of repeated measures analysis of variance are shown in Table 2.
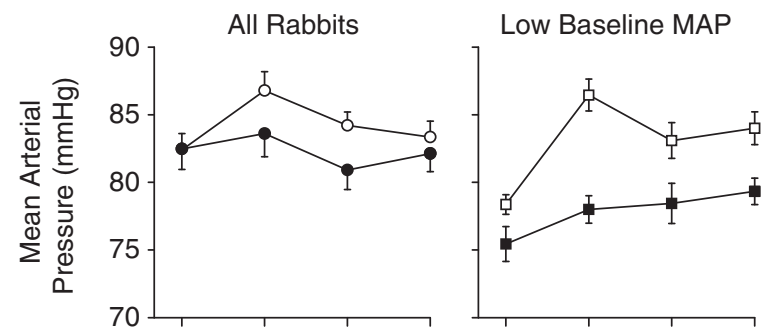

High Baseline MAP
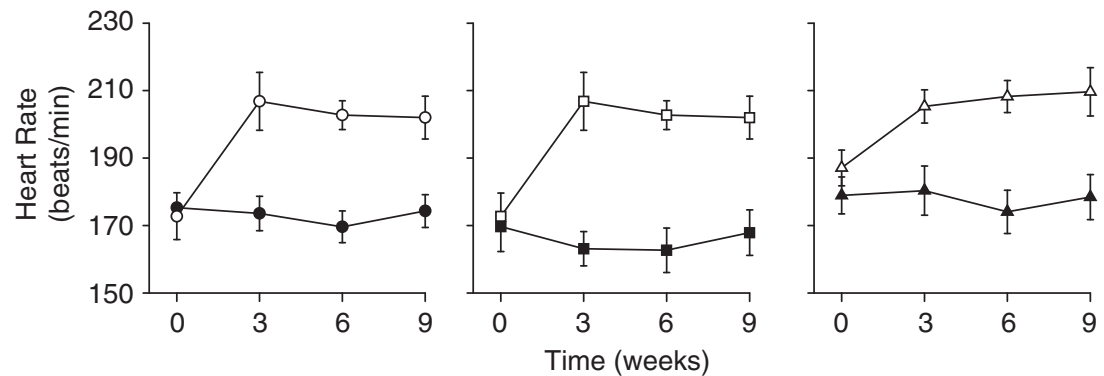

Figure 2 Mean arterial pressure (MAP) and heart rate across the 9-week dietary intervention. Data are shown for all rabbits ( $n=23$ per group), and the subgroups of rabbits with low-baseline MAP ( $\leqslant 80 \mathrm{~mm} \mathrm{Hg} ; n=9$ control and 11 fat fed) and high-baseline MAP ( $>80 \mathrm{~mm} \mathrm{Hg}, n=14 \mathrm{control}$ and 12 fat fed). Co-ordinates represent mean \pm s.e.m. Filled symbols represent rabbits on the control diet. Unfilled symbols represent rabbits on the high-fat diet. Outcomes of repeated measures analysis of variance for the complete cohort of rabbits are shown in Table 2. 


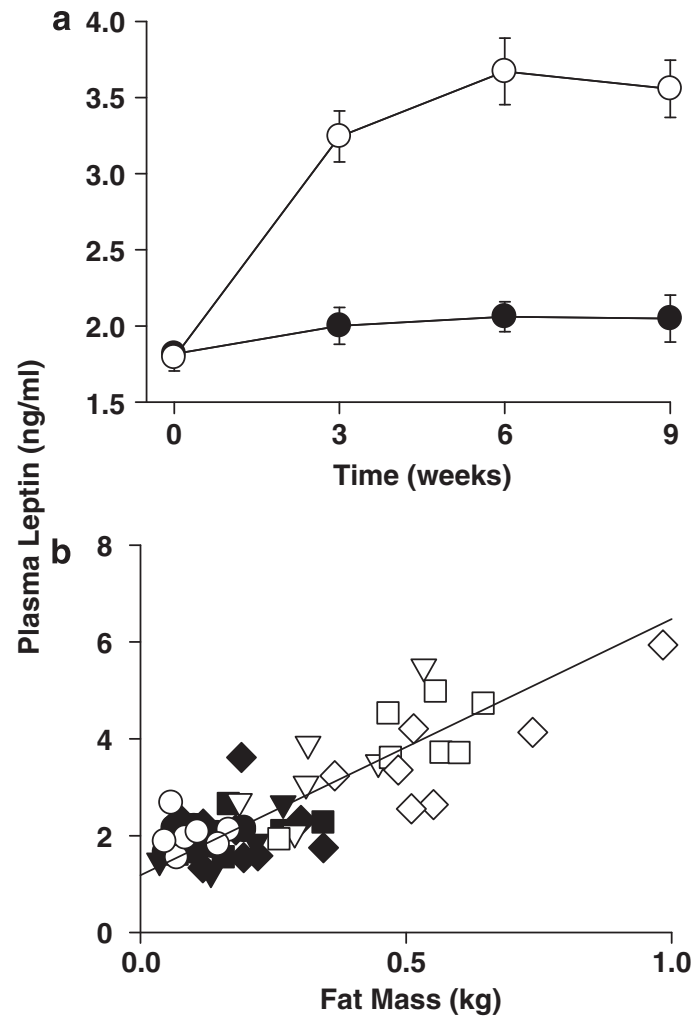

Figure 4 (a) Plasma leptin concentration across the 9-week dietary intervention. Co-ordinates represent mean \pm s.e.m. of 21 observations per group. Filled symbols represent rabbits on the control diet. Unfilled symbols represent rabbits on the high-fat diet. Outcomes of repeated measures analysis of variance are shown in Table 2. (b) Linear regression analysis of body fat vs. plasma leptin concentration. Fat mass was determined by dualenergy X-ray absorptometry. Filled symbols represent rabbits on the control diet. Unfilled symbols represent rabbits on the high-fat diet. Circles represent week 0 data, triangles represent week 3 data, rectangles represent week 6 data, diamonds represent week 9 data. Data from rabbits on contro and high-fat diets were combined for the line of best fit (solid line), determined by ordinary least-products regression analysis. ${ }^{29}$ Outcomes of regression analyses were $n=59, \quad r^{2}=0.70, \quad P<0.001, \quad X$-intercept $1.18(0.92-1.45)$, slope 5.29(4.48-6.10).

\section{Plasma creatinine and electrolyte concentrations}

Plasma creatinine concentration was less in fat-fed rabbits relative to the control rabbits from weeks 3-9 (Figure 7; Table 2), consistent with our finding of augmented glomerular filtration rate in these same fatfed rabbits under pentobarbitone anaesthesia. ${ }^{23}$ There was little systematic difference in plasma $\mathrm{Na}^{+}$or $\mathrm{Cl}^{-}$concentration between the two diet groups. Plasma $\mathrm{K}^{+}$concentration tended to be reduced in the fat-fed group relative to the control group from weeks 6-9. This may be related to elevated circulating insulin levels, as insulin is known to cause hypokalaemia. ${ }^{31}$

\section{Postmortem measurements of organ and fat pad mass}

Rabbits on the high-fat diet had significantly greater left kidney and left ventricular mass than the control group (Table 3). Although total heart mass appeared on average to be greater in the fat-fed group, this apparent effect did not reach statistical significance. When corrected for body mass, left kidney mass was not significantly different between the groups, and heart and left ventricular mass were actually
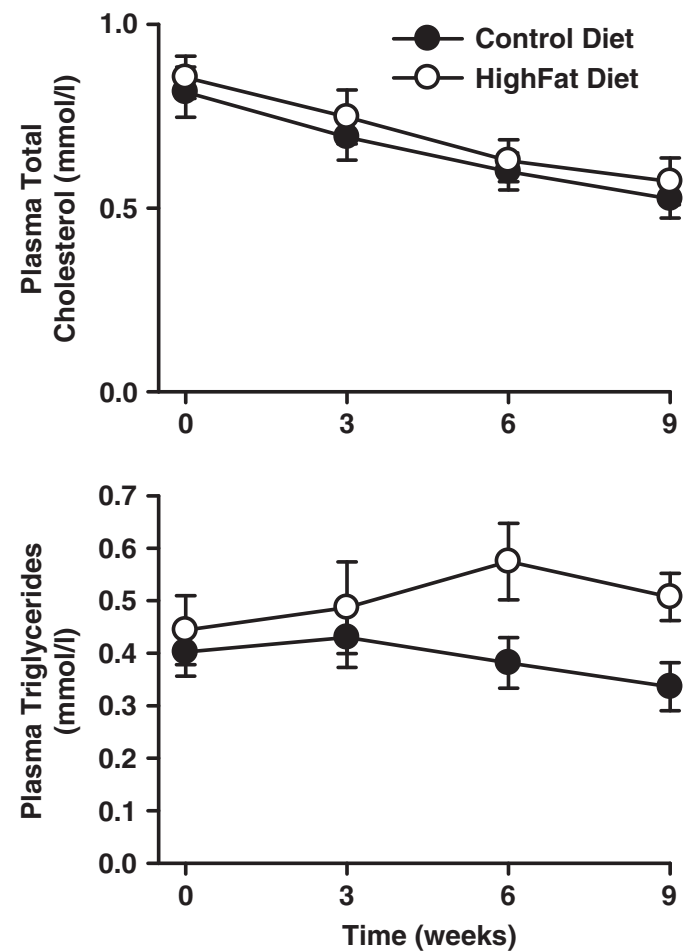

Figure 5 Plasma cholesterol and triglycerides across the 9-week dietary intervention. Co-ordinates represent mean \pm s.e.m. of $n=22$ (control diet) and 20 (high-fat diet) for total cholesterol and $n=22$ (control diet) and 21 (high-fat diet) for triglycerides. Outcomes of repeated measures analysis of variance are shown in Table 2.

less in the fat-fed group compared with the control group. Thus, there was no evidence of pathological renal or cardiac hypertrophy in fatfed rabbits. Postmortem fat pad measurements were highly correlated with total body fat determined by DEXA after 9 weeks of the dietary intervention. However, as might be expected, fat pad mass determined at postmortem greatly underestimated total fat mass as determined by DEXA (Figure 8). Fat pad mass was significantly greater for the fat-fed rabbits than control rabbits in all regions where it was measured (Table 4).

\section{DISCUSSION}

In the rabbit, fat feeding led to increased body mass, adiposity, plasma leptin levels, plasma insulin levels and HR. MAP was also elevated in rabbits with a baseline MAP below $80 \mathrm{~mm} \mathrm{Hg}$. Our data are consistent with previous studies that have shown that the pressor and tachycardic responses to fat feeding occur quickly (in a matter of days) before large increases in body mass $(<10 \%))^{9,17}$ As predicted from the results of previous studies, ${ }^{2,9,10}$ from weeks 3-9 of fat feeding, body mass and adiposity continued to increase progressively, but MAP and HR remained relatively stable. Our novel finding was that, similarly to MAP and HR, plasma leptin and insulin concentrations were also elevated at 3 weeks but stabilized thereafter. These data are consistent with the hypothesis that the absence of progression of hypertension in fat-fed rabbits can be accounted for by the absence of progression of neurohormonal abnormalities, particularly hyperleptinaemia and hyperinsulinaemia. Thus, plasma levels of leptin and insulin may be important factors in the development of hypertension and tachycardia in the rabbit model of obesity. Our observations 

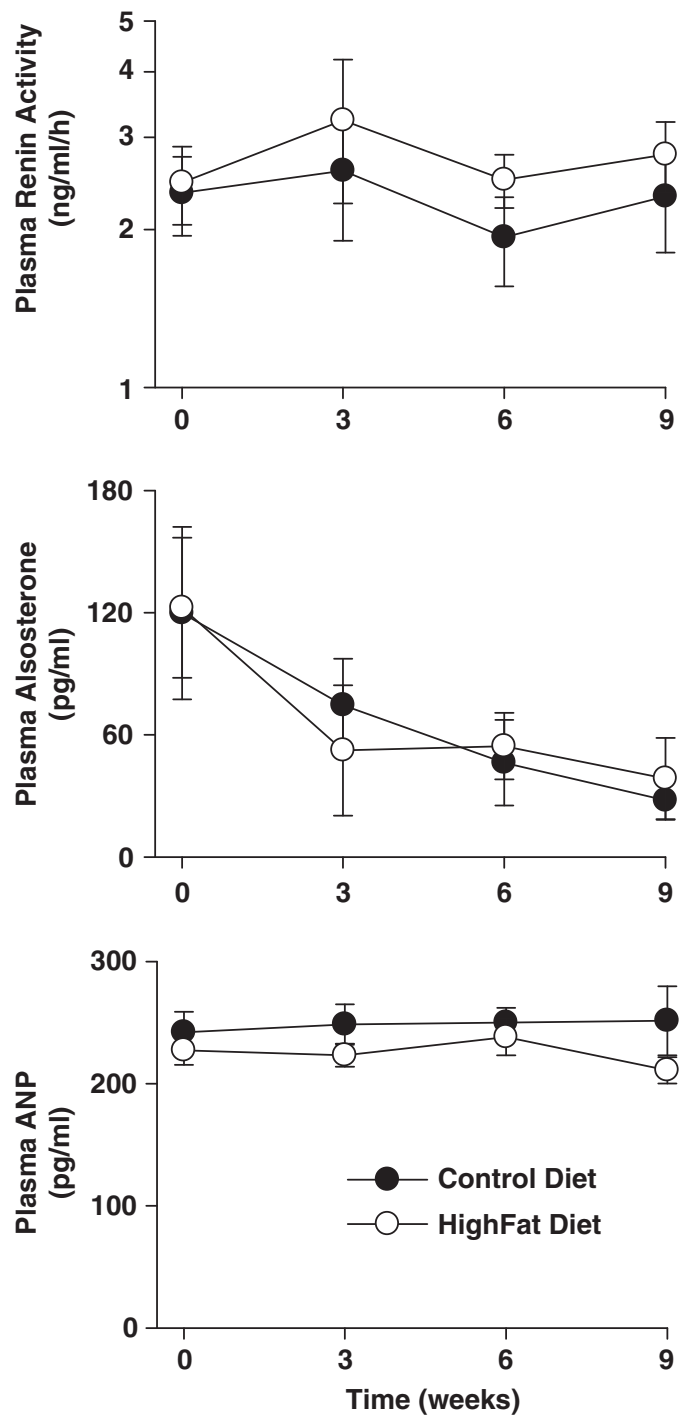

Figure 6 Plasma renin activity and aldosterone and atrial natriuretic peptide (ANP) concentrations across the 9-week dietary intervention. Co-ordinates represent mean \pm s.e.m. of $n=10$ (control diet) and 11 (high-fat diet) for plasma renin activity, and $n=6$ (control diet) and 7 (high-fat diet) for both plasma aldosterone and ANP. Outcomes of repeated measures of variance are shown in Table 2.

contrast with findings in human obesity, which demonstrate close associations between the level of adiposity as assessed by body mass index, and the degree of elevation of arterial pressure, and plasma levels of both leptin ${ }^{20-22}$ and insulin. ${ }^{32,33}$ Collectively, these data indicate that the fat-fed rabbit models the human condition well within the first 3 weeks but not in the longer term. The key difference between the human and the rabbit model may be the lack of progression, with progressive adiposity, of the neurohormonal abnormalities that drive hypertension and tachycardia. Another important difference is the apparent absence of salt-sensitivity of arterial pressure in the rabbit model. ${ }^{34}$ In contrast, human obesity is characterized by salt sensitivity. ${ }^{35}$

There is now strong evidence that hyperleptinaemia, through its ability to increase sympathetic drive, is a major driver of obesityinduced hypertension. ${ }^{36}$ Evidence for a role of hyperinsulinaemia is more equivocal. Insulin may impact on arterial pressure by increasing sympathetic drive and/or by acting as a vasodilator, at least in healthy subjects. ${ }^{37}$ Furthermore, the vasodilator action of insulin may be impaired under conditions of oxidative stress such as in the metabolic syndrome. ${ }^{38}$ Thus, the significance of hyperinsulinaemia in our rabbit model remains to be determined.

The increase in MAP we detected after 3 weeks of fat feeding was of comparable magnitude to that detected previously using New Zealand White rabbits from the same supplier and placed on identical diets to that of the current study ${ }^{16}$ and confirmed by simultaneous telemetric recordings. ${ }^{17}$ Interestingly, Armitage et al. ${ }^{17}$ recently showed that MAP, renal sympathetic nerve activity and plasma concentrations of insulin and leptin, are increased within 1 week of fat feeding. Furthermore, after 3 weeks of fat feeding, returning rabbits to the control diet resulted in normalization of plasma leptin and insulin concentrations, but renal sympathetic nerve activity and MAP remained elevated. ${ }^{17}$ Normalization of plasma leptin and insulin occurred despite maintenance of adiposity in these rabbits when they were returned to the control diet. Taken together with our current observations, over 9 weeks of fat feeding, of progressive adiposity but not progressively increased plasma concentrations of leptin or insulin, these data indicate that hyperleptinaemia and hyperinsulinaemia in fat-fed rabbits is driven chiefly by the effects of fat feeding per se rather its consequence, adiposity. However, we are also left with a paradox. That is, the findings of Armitage et al. ${ }^{17}$ and colleagues indicate that elevated MAP induced by fat feeding can be maintained in the absence of elevated plasma leptin or insulin. Yet our current findings, over a longer timescale, are consistent with the concept that the stabilization of MAP after 3 weeks of fat feeding, despite continuing progression of adiposity, could be due to the lack of progression of hyperleptinaemia and hyperinsulinaemia. While hyperleptinaemia and/or hyperinsulinaemia appear to contribute to the pro-hypertensive effects of fat feeding and adiposity, other mechanisms must also be at play.

The pressor and tachycardic response to fat feeding peaked after 3 weeks, coinciding with the time when the initial hyperphagic response to the high-fat diet was waning. Elevated plasma leptin levels likely contributed to reduced appetite across the course of the 9 weeks of fat feeding. MAP in rabbits has been shown to be strongly linked to feeding behavior. ${ }^{39}$ It seems reasonable to hypothesize, therefore, that the pressor response to fat feeding in rabbits may be in part dependent on the effects of hyperphagia and increased caloric/fat intake, and in part dependent on the chronic effects of fat feeding, both of which appear to drive hyperleptinaemia.

It is also noteworthy that 9 weeks of fat feeding, in the current study, was not associated with significant changes in PRA, or plasma levels of aldosterone or ANP. Thus, our findings provide no evidence for activation of the renin-angiotensin-aldosterone system in the fatfed rabbits we studied.

We also found that the blood pressure response to fat feeding depended on baseline MAP. Thus, a pressor response to fat feeding, of similar magnitude to that observed in previous studies, ${ }^{2,6-17}$ was seen in rabbits with a baseline MAP $\leqslant 80 \mathrm{~mm} \mathrm{Hg}$, but not in rabbits with a baseline MAP $>80 \mathrm{mmHg}$. This finding confirms our anecdotal observations from previous studies.

The strengths of our current study include the relatively large sample size of fat-fed and lean rabbits $(n=23$ each) and the fact that we were able to follow these rabbits serially across a 9-week dietary intervention. Our experiment also validated, against the gold standard of dual X-ray absorptometry, the use of postmortem dissection of fat pads as an index of total adiposity in rabbits. 

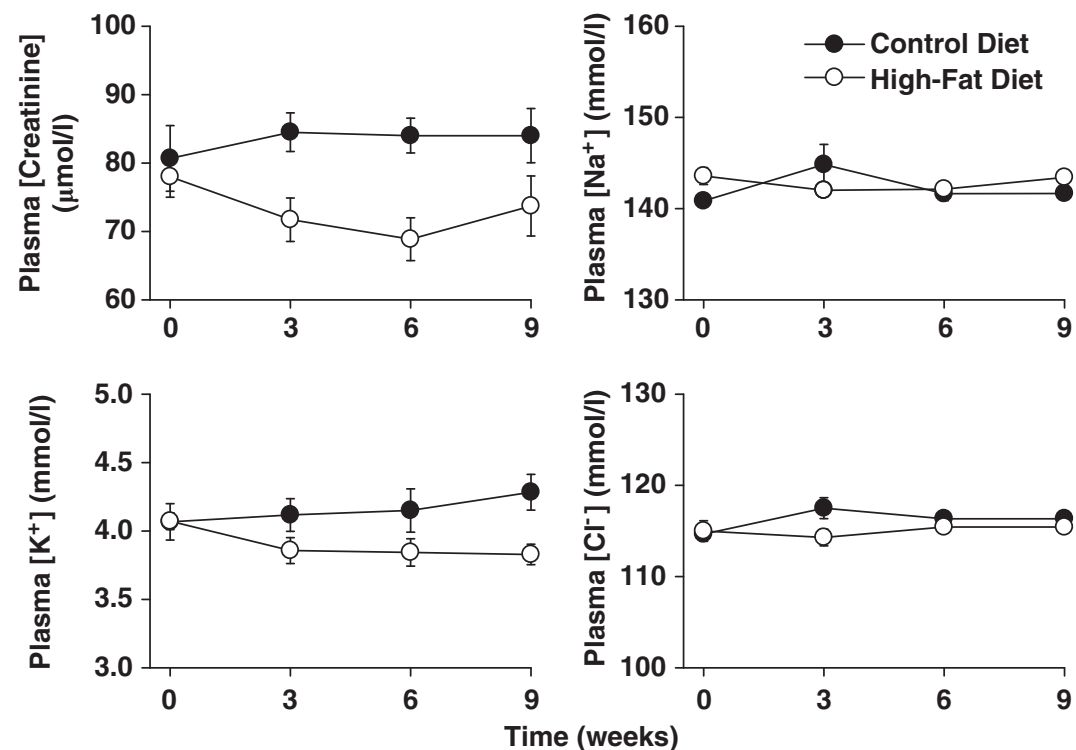

Figure 7 Plasma creatinine and electrolytes across the 9-week dietary intervention. Co-ordinates represent mean \pm s.e.m. of $n=6$ (control diet) and 7 (highfat diet) for all four variables. Outcomes of repeated measures analysis of variance are shown in Table 2.

Table 3 Organ mass measured at postmortem

\begin{tabular}{|c|c|c|c|c|c|c|}
\hline \multirow[b]{2}{*}{ Diet } & \multicolumn{2}{|c|}{ Left kidney mass } & \multicolumn{2}{|c|}{ Heart mass } & \multicolumn{2}{|c|}{ Left ventricular mass } \\
\hline & (g) & (g kg-1 body mass) & (g) & (g kg-1 body mass) & (g) & (g kg-1 body mass) \\
\hline High fat & $12.0 \pm 0.3^{* *}$ & $2.77 \pm 0.08$ & $8.4 \pm 0.2$ & $2.01 \pm 0.04^{*}$ & $5.1 \pm 0.1^{*}$ & $1.21 \pm 0.03^{*}$ \\
\hline
\end{tabular}

Data are presented as mean \pm s.e.m. $(n=23)$.

${ }^{*} P<0.05,{ }^{* *} P<0.01$ for comparisons between rabbits on the two diets, from Student's unpaired $t$-test.

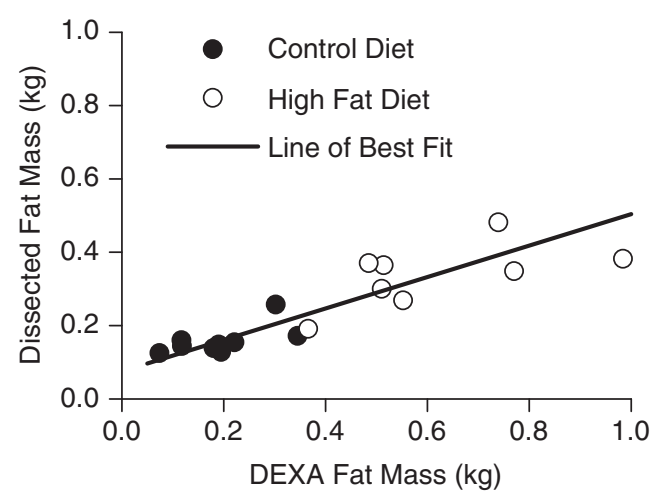

Figure 8 Linear regression analysis of fat mass determined by dual-energy X-ray absorptometry (DEXA) vs. that determined by dissection at postmortem. Data from rabbits on control and high-fat diets were combined for the line of best fit, determined by ordinary least-products regression analysis. ${ }^{29}$ Outcomes of regression analysis are as follows $n=17$, $r^{2}=0.771, \quad P<0.001, \quad X$-intercept $=0.08(0.02-0.13), \quad$ slope $=0.43$ (0.31-0.055).

In conclusion, our study documented an experimental model of progressive obesity in rabbits in which the increase in arterial pressure peaks at 3 weeks, despite progressive development of adiposity over the 9 weeks of fat feeding. The fact that the increase in MAP in
Table 4 Mass of fat pads dissected at postmortem

\begin{tabular}{lccccc}
\hline Diet & Peri-cardial (g) & Gonadal (g) & Peri-renal (g) & Mesenteric (g) & Total (g) \\
\hline Control & $9.0 \pm 0.6$ & $8.1 \pm 0.5$ & $74 \pm 5$ & $69 \pm 6$ & $160 \pm 11$ \\
High fat & $14.8 \pm 0.7^{* * *}$ & $18.2 \pm 1.5^{* * *}$ & $154 \pm 10^{* * *}$ & $133 \pm 9^{* * *}$ & $319 \pm 19^{* * *}$ \\
\hline
\end{tabular}

Data are presented as mean \pm s.e.m. ${ }^{* *} P<0.001$.

this model does not progress beyond 3 weeks of fat feeding suggests that this model is not ideal for studying the long-term effects of adiposity but better mimics the short-term effects of fat feeding. Our findings also show that the development of obesity-related hypertension is dependent on the resting MAP before the instigation of a high-fat diet.

\section{CONFLICT OF INTEREST}

The authors declare no conflict of interest.

\section{ACKNOWLEDGEMENTS}

This work was supported by grants from the National Health and Medical Research Council of Australia (384101) and the National Heart Foundation of Australia (G08M3810). The study was supported in part by the Victorian Government's Operational Infrastructure Support Program. 
1 Kshatriya S, Reams GP, Spear RM, Freeman RH, Dietz JR, Villarreal D. Obesity hypertension: the emerging role of leptin in renal and cardiovascular dyshomeostasis. Curr Opin Nephrol Hypertens 2010; 19: 72-78.

2 Antic V, Van Vliet BN, Montani J-P. Loss of nocturnal dipping of blood pressure and heart rate in obesity-induced hypertension in rabbits. Autonomic Neurosci Basic Clin 2001; 90: 152-157.

3 Verwaerde P, Senard JM, Galinier M, Rouge P, Massabuau P, Galitzky J, Berlan M, Lafontan M, Montastruc JL. Changes in short-term variability of blood pressure and heart rate during the development of obesity-associated hypertension in high-fat fed dogs. J Hypertens 1999; 17: 1135-1143.

4 Celoria BM, Genelhu VA, Pimentel Duarte SF, Delfraro PA, Francischetti EA. Hypoadiponectinemia is associated with prehypertension in obese individuals of multiethnic origin. Clin Cardiol 2010; 33: E61-E65.

5 Rizvi AA. Hypertension, obesity, and inflammation: the complex designs of a deadly trio. Metab Syndr Relat Disord 2010; 8: 287-294.

6 Carroll JF, Huang M, Hester RL, Cockrell K, Mizelle HL. Hemodynamic-alterations in hypertensive obese rabbits. Hypertension 1995; 26: 465-470.

7 Carroll JF, Dwyer TM, Grady AW, Reinhart GA, Montani JP, Cockrell K, Meydrech EF, Mizelle HL. Hypertension, cardiac hypertrophy, and neurohumoral activity in a new animal model of obesity. Am J Physiol Heart Circ Physiol 1996; 271: H373-H378.

8 Dwyer TM, Carroll JF, Mizelle HL, Cockrell K. Renal size and composition in hypertensive, obese rabbits. Int J Obesity 1998; 22: 935-938.

9 Antic V, Tempini A, Montani J-P. Serial changes in cardiovascular and renal function of rabbits ingesting a high-fat, high-calorie diet. Am J Hypertens 1999; 12: 826-829.

10 Antic V, Kiener-Belforti F, Tempini A, Van Vliet BN, Montani J-P. Role of the sympathetic nervous system during the development of obesity-induced hypertension in rabbits. Am J Hypertens 2000; 13: 556-559.

11 Carroll JF, Kyser CK. Exercise training in obesity lowers blood pressure independent of weight change. Med Sci Sports Exerc 2002; 34: 596-601.

12 Carroll JF, King JW, Cohen JS. Hydralazine as antihypertensive therapy in obesityrelated hypertension. Int J Obes 2004; 28: 384-390.

13 Carroll JF, King JW, Wright AM, Thaden JJ. Effect of obesity and hydralazine treatment on diurnal rhythms of blood pressure and heart rate. J Mol Cell Cardiol 2004; 37: 234-234.

14 Carroll JF, Thaden JJ, Wright AM, Strange T. Loss of diurnal rhythms of blood pressure and heart rate caused by high-fat feeding. Am J Hypertens 2005; 18 : 1320-1326.

15 Carroll JF, Thaden JJ, Wright AM. A comparison of two exercise training programs on cardiac responsiveness to beta-stimulation in obesity. Exp Biol Med 2005; 230: 180-188.

16 Prior LJ, Eikelis N, Armitage JA, Davern PJ, Burke SL, Montani JP, Barzel B, Head GA. Exposure to a high-fat diet alters leptin sensitivity and elevates renal sympathetic nerve activity and arterial pressure in rabbits. Hypertension 2010; 55: 862-868.

17 Armitage JA, Burke SL, Prior LJ, Barzel B, Eikelis N, Lim K, Head GA. Rapid onset of renal sympathetic nerve activation in rabbits fed a high-fat diet. Hypertension 2012; 60: $163-171$.

18 Doll S, Paccaud F, Bovet P, Burnier M, Wietlisbach V. Body mass index, abdominal adiposity and blood pressure: consistency of their association across developing and developed countries. Int J Obes Relat Metab Disord 2002; 26: 48-57.

19 Kotsis V, Stabouli S, Bouldin M, Low A, Toumanidis S, Zakopoulos N. Impact of obesity on 24-hour ambulatory blood pressure and hypertension. Hypertension 2005; 45: 602-607.
20 Barba G, Russo O, Siani A, lacone R, Farinaro E, Gerardi MC, Russo P, Della Valle E, Strazzullo P. Plasma leptin and blood pressure in men: graded association independent of body mass and fat pattern. Obes Res 2003; 11: 160-166.

21 Matsubara M, Chiba H, Maruoka S, Katayose S. Elevated serum leptin concentrations in women with components of multiple risk factor clustering syndrome. $J$ Atheroscler Thromb 2000; 7: 231-237.

22 Nakamura Y, Ueshima H, Okuda N, Murakami Y, Miura K, Kita Y, Okamura T, Turin TC, Rodriguez B, Curb JD, Stamler J. Relation of serum leptin to blood pressure of Japanese in Japan and Japanese-Americans in Hawaii. Hypertension 2009; 54: 1416-1422.

23 Eppel GA, Head GA, Denton KM, Evans RG. Effects of tempol and candesartan on neural control of the kidney. Autonom Neurosci 2012; 168: 48-57.

24 Michaels S, Eppel GA, Burke SL, Head GA, Armitage J, Carroll JF, Malpas SC, Evans RG. Altered responsiveness of the kidney to activation of the renal nerves in fat-fed rabbits. Am J Physiol Regul Integr Comp Physiol 2009; 296: R1889-R1896.

25 Johnston $\mathrm{Cl}$, Mendelsohn F, Casley D. Evaluation of renin and angiotensin assays and their clinical application. Med J Aust 1971; 1: 126-128.

26 Evans RG, Burke SL, Lambert GW, Head GA. Renal responses to acute reflex activation of renal sympathetic nerve activity and renal denervation in secondary hypertension. Am J Physiol Regul Integr Comp Physiol 2007; 293: R1247-R1256.

27 Chen H, Sullivan G, Yue LQ, Katz A, Quon MJ. QUICKI is a useful index of insulin sensitivity in subjects with hypertension. Am J Physiol Endocrinol Metab 2003; 284: E804-E812.

28 Ludbrook J. Repeated measurements and multiple comparisons in cardiovascular research. Cardiovasc Res 1994; 28: 303-311.

29 Ludbrook J. Linear regression analysis for comparing two measurers or methods of measurement: but which regression? Clin Exp Pharmacol Physiol 2010; 37: 692-699.

30 Drummond GB, Tom BDM. Statistics, probability, significance, likelihood: words mean what we define them to mean. Clin Exp Pharmacol Physiol 2011; 38: 555-558.

31 Clausen T. Hormonal and pharmacological modification of plasma potassium homeostasis. Fundam Clin Pharmacol 2010; 24: 595-605.

32 Modan M, Halkin H, Almog S, Lusky A, Eshkol A, Shefi M, Shitrit A, Fuchs Z. Hyperinsulinemia. A link between hypertension obesity and glucose intolerance. J Clin Invest 1985; 75: 809-817.

33 Ferrannini E, Mari A. Beta cell function and its relation to insulin action in humans: a critical appraisal. Diabetologia 2004; 47: 943-956.

34 Antic V, Kiener-Belforti F, Tempini A, Montani J-P. Obesity-induced hypertension in rabbits is not salt-sensitive. J. Hypertens 1999; 17 (Suppl 3): S209 (Abstract).

35 Rocchini AP, Key J, Bondie D, Chico R, Moorehead C, Katch V, Martin M. The effect of weight loss on the sensitivity of blood pressure to sodium in obese adolescents. N Engl J Med 1989; 321: 580-585.

36 Hall JE, Hildebrandt DA, Kuo J. Obesity hypertension: role of leptin and sympathetic nervous system. Am J Hypertens 2001; 14: 103S-115S.

37 Anderson EA, Hoffman RP, Balon TW, Sinkey CA, Mark AL. Hyperinsulinemia produces both sympathetic neural activation and vasodilation in normal humans. J Clin Invest 1991; 87: 2246-2252.

38 Schinzari F, Tesauro M, Rovella V, Galli A, Mores N, Porzio O, Lauro D, Cardillo C. Generalized impairment of vasodilator reactivity during hyperinsulinemia in patients with obesity-related metabolic syndrome. Am J Physiol Endocrinol Metab 2010; 299: E947-E952.

39 Van Den Buuse M, Malpas SC. 24-hour recordings of blood pressure, heart rate and behavioural activity in rabbits by radio-telemetry: Effects of feeding and hypertension. Physiol Behav 1997; 62: 83-89. 